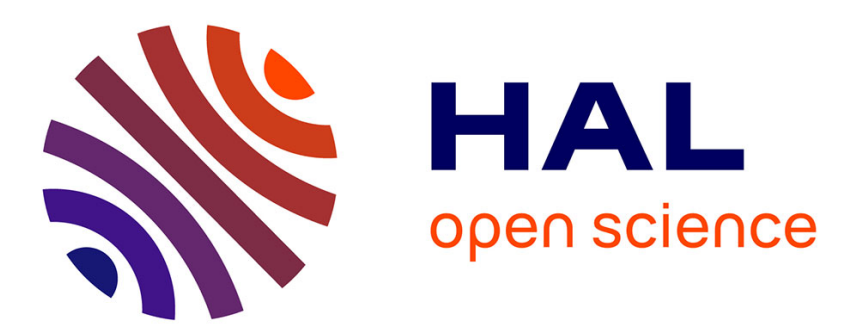

\title{
Remarques sur le fragment de Cambridge du Journal des rêves de Myōe (1173-1232) \\ Frédéric Girard
}

\section{To cite this version:}

Frédéric Girard. Remarques sur le fragment de Cambridge du Journal des rêves de Myōe (1173-1232). Bulletin de l'Ecole française d'Extrême-Orient, 1999, 86 (1), pp.377-384. 10.3406/befeo.1999.3419 . hal-02533565

\section{HAL Id: hal-02533565 https://hal.science/hal-02533565}

Submitted on 6 Apr 2020

HAL is a multi-disciplinary open access archive for the deposit and dissemination of scientific research documents, whether they are published or not. The documents may come from teaching and research institutions in France or abroad, or from public or private research centers.
L'archive ouverte pluridisciplinaire HAL, est destinée au dépôt et à la diffusion de documents scientifiques de niveau recherche, publiés ou non, émanant des établissements d'enseignement et de recherche français ou étrangers, des laboratoires publics ou privés. 


\section{Remarques sur le fragment de Cambridge du Journal des rêves de} Myōe (1173-1232)

Frédéric Girard

\section{Résumé}

Abstract Some Remarks on the Harvard Fragment of the Dreams Record of Myôe (1173-1232) In the present paper, I analyse a fragment of the Dream Diary of Myôe (1173-1232), conserved at the Harvard University Art Museum in Cambridge, Mass. This fragment seems to be a better, that is earlier, version of the Dream Diary than the one that has been conserved in Kôzanji monastery, and that has already been published. Here we present a new translation and analysis of it. I refer to the hypothesis concerning the identification of the personnage of Ko no sanmi no tsubone, who seems to be alluded to in this fragment, and who is a great patron of Myôe and Kôzanji. This identification is not only interesting from the historical point of view, but also provides new perspectives on the relationship between Myôe, traditionally considered as representative of ancient Buddhism, and Dôgen, who is thought to be representative of the so-called new Buddhism of this period : in fact, both communities seem to have had the same patron. The discovery of historical details permits us to reconsider pertinent paradigms and categories to define and analyse medieval Buddhism as japanised Buddhism.

\section{Citer ce document / Cite this document :}

Girard Frédéric. Remarques sur le fragment de Cambridge du Journal des rêves de Myōe (1173-1232). In: Bulletin de l'Ecole française d'Extrême-Orient. Tome 86, 1999. pp. 377-384;

doi : https://doi.org/10.3406/befeo.1999.3419

https://www.persee.fr/doc/befeo_0336-1519_1999_num_86_1_3419

Fichier pdf généré le 08/02/2019 


\section{Remarques sur le fragment de Cambridge du Journal des rêves de Myōe (1173-1232)}

\section{Frédéric GIRARD*}

L'existence d'un fragment du Journal des rêves de Myōe, conservé au Harvard University Art Museum de Cambridge (Mass.), aux États-Unis, nous a été obligeamment communiquée par M. Sylvan Barnet. Il s'agit d'un fragment provenant du pavillon du Hōbenchiin, le centre d'études du Kōzanji, dont il porte l'estampillage. Il se présente sous la forme d'un rouleau suspendu de $33,7 \times 55,3 \mathrm{~cm}$.

Il rapporte des rêves faits par Myōe le 16 du $8^{\mathrm{e}}$ mois de 1225 et correspond à un fragment du Journal de Myōe conservé au Kōzanji, qui est une copie datant du milieu de l'époque Kamakura de la main d'un scribe non identifié avec certitude; on peut néanmoins supposer qu'il s'agit de Jōshin (1174-1250), un des principaux disciples de Myōe en ésotérisme ${ }^{1}$. Il est toutefois plus long de quelques lignes que ce dernier et comporte des variantes de lecture. Étant donné que le fragment de Cambridge [A] semble bien être de la main de Myōe, on peut supposer que le fragment du Kōzanji [B] est soit une copie tronquée assez lointaine et assez peu adroite du premier, soit reproduit une autre version encore, qui ne nous est pas parvenue.

C'est cette deuxième supposition qui paraît la plus plausible, si l'on veut bien prendre en considération le fait qu'existe, au moins, une différence de graphisme que peut difficilement expliquer une simple erreur de copiste ou une dissemblance de style calligraphique : celle qui vient clore [B], fusu tokoro 伏 ス所, "être allongé ou couché ", se trouve pour aru issho 或一所, 《en un certain endroit », dans [A]. La version [A] est d'ailleurs dans son ensemble beaucoup plus cohérente et satisfaisante que la version [B]. C'est donc sur elle qu'il faut se fonder pour lire le rêve en question, dont je présente la traduction; c'est aussi pour moi une occasion de remettre de cette façon à jour la traduction que $\mathrm{j}$ 'avais précédemment présentée.

Le présent fragment rapporte des visions faites par Myōe au moment où l'on a inauguré au Kōzanji les sanctuaires de trois divinités, Byakkō, Zenmyō et Kasuga, représentant respectivement l'Inde, la (Chine/)Corée et le Japon, et qu'incarnent trois personnages humains proches de Myōe, le moine Dōchū (1178-1223), une cousine Maya (?-?), et un ami poète Fujiwara no Chikayasu (?-?). L'existence de plusieurs versions connues de ces rêves - outre [A] et [B], la longue relation du Journal de Kikai à l'âge de 48 ans [C], et peut-être d'autres encore - n'a pas de quoi nous étonner : elle montre simplement l'importance que devait avoir l'événement dans la conscience de leurs

* Membre de l'École française d'Extrême-Orient.

1. Il s'agit du fragment $\mathrm{n}^{\circ} 30$ de notre liste ; cf. Frédéric Girard, Un moine de la secte Kegon à l'époque de Kamakura, Myōe (1173-1232) et le Journal de ses rêves, Paris, École française d'Extrême-Orient (PEFEO, 160), $1990: 115-116$; trad. ibid. : 194-195, rêve ${ }^{\circ} 237$; transcription Okuda Isao, MSS, II : 501. 
instigateurs ainsi que pour la communauté monacale. Une vision comme celle de la montée au ciel Tușita ${ }^{2}$, par exemple, est notée non seulement par Myōe lui-même deux fois au moins dans des recueils différents, mais encore par ses biographes ainsi que par un " témoin » extérieur comme l'a été Gyōhen (1181-1264), un moine du Ninnaji; on pourrait en dire autant de la vision qu'il a eue du bodhisattva Mañjuśri ${ }^{3}$. Son entourage immédiat et sa postérité les rapportent avec la vénération respectueuse qui est due à un témoignage considéré comme indubitable, qu'en ces circonstances tel enseignement ou telle règle ont été effectivement transmis de haute autorité.

Pour le fragment qui nous occupe, il est précisément question d'un texte de préceptes religieux apparemment destiné aux communautés du Kōzanji, pour les religieux, et du Zenmyōji, pour les moniales, encore que rien ne nous dise comment ni dans quelles circonstances ni par qui il a été transmis. On peut tout du moins remarquer que la divinité Byakkō, sous les traits du préfet monacal Dōchū, se pose dans le rêve en maître de prédication et des préceptes de la communauté de moniales du Zenmyōji, rôle qu'elle est d'autant mieux placée à jouer qu'elle est, selon les textes, réputée enseigner des méthodes de méditation ${ }^{4}$; le personnage de Zenmyō représente par excellence la dévote laïque ou la moniale pieuse, et celui de la divinité de Kasuga, l'emblême des laïcs de la famille des Fujiwara. Myōe reproduit à ses fidèles l'image de leur propre idéal.

Ce fragment, en situant une des scènes de ces rêves dans la résidence du Gouverneur de troisième rang, pose par ailleurs incidemment la question de savoir qui était la Dame du commandant de la garde des portes de troisième rang, dont font état les sources du Kōzanji, et qui s'est trouvé particulièrement liée à Myōe ainsi qu'aux édifications religieuses du Zenmyōji et du Kōzanji, après les guerres civiles de Jōkyū $(1221)^{5}$. On devine en effet ici sa présence comme en filigrane, s'agissant de la dédicace de statues, où on la voit à l'occasion intervenir en tant que commanditaire. La question de son identification n'est pas sans quelque intérêt, sur au moins deux plans que je ne ferai qu'évoquer ici : d'une part, en sachant à quels milieux et à quels personnages Myōe avait affaire dans le cadre de ses activités religieuses, c'est la signification même de celles-ci qui s'en trouve éclairée - s'est-il fait le défenseur de la veuve et de l'orphelin ? a-t-il pris le parti de la cour impériale de Kyōto ou des militaires de Kamakura ? était-il un réformateur ou un conservateur, ou s'est-il défini autrement encore? D'autre part, la connaissance exacte des personnages laïcs entourant Myōe peut nous ouvrir des perspectives sur les liens qu'il entretenait avec d'autres religieux, sur le plan des doctrines, des pratiques cultuelles et méditatives, de la circulation des livres et des disciples, ainsi que sur la poésie - je pense aux rapports directs ou indirects que Myōe a pu avoir avec de célèbres contemporains, comme Shunjō (1166-1227) ou Dōgen (1200-1253) -, en raison du fait que ces religieux évoluent dans des milieux qui se jouxtent sans que l'on sache s'ils se connaissaient ou s'ignoraient mutuellement, bien qu'on ait pu constater entre eux des similitudes, des affinités ou parfois des oppositions dans leurs attitudes.

Concernant le premier point, on a proposé deux identifications raisonnables pour le personnage de la Dame du commandant de la garde des portes du troisième rang (Kō no sanmi no tsubone), qui est la commanditaire d'œuvres d'art du Kōzanji, comme le célèbre rouleau peint sur l'Historique de l'école de l'Ornementation fleurie (Kegon engi). On a pensé à Fujiwara no Kishi (?-?), qui est une sœur de Shumeimon.in (1182-1264), la

2. Rêve $\mathrm{n}^{\circ} 104$.

3. Rêve $n^{\circ} 1$.

4. F. Girard, Un moine..., op. cit., note 1206.

5. En 1217-1218 (Historique du Közanji, Közanji engi, MSS, I : 650 et 677), en 1219, $11^{\mathrm{e}}$ mois, $11^{\mathrm{e}}$ jour (idem : 636), en 1223 (idem : 657). 
favorite de l'empereur Gotoba, et qui a donné naissance à l'empereur Juntoku (1197$1242)$, et est une fille de Fujiwara no Norisue $(1138-1205)^{6}$. On a avancé également le nom de Jishi (?-?), une de ses cousines, née d'un mariage entre Fujiwara Hanshi (?-1200) et le moine Nōen (?-1185), un fils adoptif de Taira no Kiyomori (1118-1181); Jishi est sœur utérine de l'épouse impériale Shōmeimon.in (1171-1257), qui est elle-même mère de l'empereur Tsuchimikado (1195-1231), et connue comme Jishi «fille adoptive » de Minamoto no Michichika (1149-1202) ${ }^{7}$. L'argument majeur mis en avant pour plaider pour la seconde hypothèse est que, d'un mariage de Jishi avec Fujiwara no Tadasue (11301196), lui serait né Chikahira (1196-1213), mort prématurément, et que d'un autre avec Konoe no Motomichi (1160-1233), serait issue la moniale Zenni ; or, ces deux enfants sont mentionnés par les sources du Kōzanji, et les événements les concernant sont en congruence avec l'apparition de la Dame du commandant du troisième rang dans l'horizon de Myōe.

Toutefois, les imprécisions et les lacunes des lignées généalogiques, comme celles du Sonpi bunmyaki (XIV ${ }^{\mathrm{e}}$ siècle) auxquelles on se réfère, ne sont pas sans faire peser des doutes sur les hypothèses les mieux étayées : ne font-elles pas, par exemple, de Shumeimon.in à la fois la sœur et la fille de Norisue $^{8}$, et de Chikahira le fils de Jishi et d'une sœur cadette de celle-ci ${ }^{9}$ ? En outre, Jishi elle-même y est présentée comme épouse de Tadasue, puis du kanpaku Tadamichi (1097-1164), le grand-père de Motomichi, ce qui est impossible. On pourrait de fait penser à d'autres candidates, sans exclusive, d'autant que le titre de Dame du commandant de la garde des portes du troisième rang peut désigner aussi bien une épouse qu'une sœur ou qu'une fille d'un personnage portant le titre de commandant du troisième rang, ce qui accroît le nombre des possibilités. Néanmoins, je retiendrai actuellement le nom de Jishi comme étant le plus probable, en raison du contexte historique.

Concernant le second point, ce sont en particulier des questions relatives aux pratiques méditatives et aux règles disciplinaires nouvellement venues de Chine qui se posent à propos de ces personnages religieux. Les rapports entre Shunjō et Dōgen sont avérés, puisqu'ils sont ceux de maître à disciple ; ils se sont tissés au cours d'une période qu'on peut situer entre 1215/1217 et $1221^{10}$. Ceux entre Shunjō et Myōe sont probables, quoiqu'une rencontre entre les deux hommes ne soit pas certaine ${ }^{\prime \prime}$. Enfin ceux entre Myōe et Dōgen sont possibles, au moins indirectement, quoique non avérés ; plusieurs éléments plaident en leur faveur, que je me contente ici de mentionner: une visite possible de Dōgen à Takao en 1208 après la disparition de sa mère, où il aurait réalisé l'« impermanence de ce qui naît et disparaît », site lié au jeune Myōe ainsi qu'au nom de Mongaku (1138-1204) que Minamoto no Michichika, personnage qui fut considéré

6. Okuda Isao, Myōe, itinéraire et rêve (Myōe - henreki to yume), Tōkyō daigaku shuppankai, 1978 : 191 . Cf. F. Girard, op. cit., notes 615 et 821.

7. Karen L. Brock, "Chinese Maiden, Silla Monk : Zenmyō and Her Thirteenth-Century Japanese Audience ", in Flowering in the Shadows, Women in the History of Chinese and Japanese Painting, Marsha Weidner éd., Honolulu, University of Hawaii Press, $1990: 207-209$; et Karen L. Brock, Tales of Gisho and Gangyo : Editor, Artist, and Audience in Japanese Picture Scrolls, Princeton University, Ph.D. dissertation, 1984, University Microfilms international : 368-374.

À la mort de sa mère, Hanshi, survenue en 1200 , Shōmeimon.in est en outre devenue épouse de Michichika qui l'a choisie en remplacement. Le titre de « fille adoptive» ne doit pas faire illusion.

8. Sonpi bunmyaku, II : 478 et $482-483$.

9. Ibidem, I : 205 et II : 139.

10. Nōtomi Jōten, Recherches sur les matériaux de la bibliothèque de Kanazawa (Kanazawa bunko shiryō no kenkyūu), Kyōto, Hōzōkan, $1983:$ 529-540.

11. F. Girard, op. cit. : XXXVIII-XL et note 24. 
comme étant le père de Dōgen mais qui fut plus vraisemblablement son beau-père ou son grand-père, aurait pu faire exiler en $1199^{12}$; l'inauguration sous le patronage d'un fidèle du shōgun Minamoto no Sanetomo (1192-1219), Ganshō (Kuzuyama Gorō Kagenori) (?-?), le $15 \mathrm{du} 10^{\mathrm{e}}$ mois de 1227, du monastère Saihōji de Yura en compagnie (?) de Myōe, du Sceau de la Loi Myōzen (1167-1242), supérieur du pavillon de Bishamon au Hieizan, ainsi que d'autres personnages, a été faite avec un panonceau d'entrée dont le nom - Saihōji - a été fixé par Myōe et dont on aurait demandé la calligraphie à Dōgen qui venait semble-t-il juste de rentrer de Chine ${ }^{13}$; une visite de Myōe à Kobata, pour donner l'extrême-onction à une fille adoptive de Motofusa (1145-1230), personnage qui passe pour être père adoptif de Dōgen, le 13 du $9^{\mathrm{c}}$ mois de $1230^{14}$; une circulation avérée de moines de l'école de Bodhidharma chez Dōgen et Myōe, comme chez d'autres moines d'ailleurs.

Ajoutons qu'une étude récente sur la biographie de Dōgen tient que l'hypothèse la plus probable pour identifier la moniale appelée Shōgakuni qui a protégé Dōgen durant la période où il a vécu à Fukakusa (vers 1235-1243), près de Kyōto, est précisément Jishi dont on vient de parler, qui est épouse en deuxièmes noces de Konoe Motomichi (1160-

12. Selon la biographie de Dōgen rédigée par Keizan (1266-1325), Recueil de la transmission de la lumière (Denkōroku 傳光錄, T. 82, $\mathrm{n}^{\circ} 2585$ : 405ab).

Il semblerait, selon une étude faite à partir des documents du Jingoji, que Mongaku a été arrêté par la police le $2^{\mathrm{e}}$ mois de 1199 (Shöji, 1), en raison d'un complot ourdi contre lui par Minamoto no Michichika, avant que ne soit prononcé son exil à Sado le 17 du mois suivant. Je n'ai pu avoir accès à ces documents, mais il y est fait référence dans l'article de Tai Keigo, « $\grave{A}$ propos des manuscrits du Jingoji (Jingoji monjo ni tsuite) », in Shirin, 25 (1), 1940:91-92, lettre n 274 ; voir Azuma Ryūshin, Petit dictionnaire sur Dōgen (Dōgen shōjiten), Tōkyō, Shunjūsha, 1982: 8-9. Le fait est loin d'être impossible, car Michichika ainsi que son épouse, Shumeimon.in, jouaient à cette époque, depuis 11961198 notamment, un rôle central dans la manière dont était conduite la politique, aux dépens des membres de la famille Kūjō et tandis que l'empereur Gotoba (1180-1239) venait de «faire retraite» (Tomikura Tokujirō, Commentaire complet au Dit des Heike (Heike monogatari zenchūshaku), Kadokawa shoten, Tōkyō, $1970: 146-151$ ). Toutefois, l'opinion prévaut chez plusieurs auteurs que c'est l'empereur Gotoba lui-même qui a directement destitué Mongaku au profit de ses proches (Yamada Shōzen, «Étude sur la chronologie abrégée du religieux Mongaku [Sō Mongaku ryakunenpu-kō] », in Rikkyō daigaku Nihon bungaku, Tōkyō, 1964: 17; Aihara Seiji, Chronique de la vie du religieux Mongaku [Mongaku shōnin ichidaiki], Seiabō, Tōkyō, 1985 : 225-236). Ne peut-on supposer que c'est, en réalité, Michichika qui a fait agir Gotoba ? En effet, Mongaku est revenu à Takao après la mort de Michichika en 1202.

13. «Chronologie des actes du maître national Hattō Enmyō, fondateur du Pic aux Vautours (Washigamine kaisan Hattō Enmyō kokushi gyōjitsu nenpu) », ZGSRJ, 9: 348-349. Voir Ōkubo, Recherches sur la biographie du maître de dhyāna Dōgen (Dōgen zenji den no kenkyū), Iwanami shoten, Tōkyō, 1953 : 195-196. Ce fait en particulier incline à penser que Dōgen et Myōe se connaissaient au moins de nom dès cette époque. La similitude du rôle assigné au temple du Saihōji et du Eiheiji, à savoir prier pour l'âme du shōgun assassiné Sanetomo (1192-1219) et pour le salut posthume de sa mère Hōjō Masako (1157-1225), donne du crédit à l'idée que Dōgen a participé à l'inauguration de ce monastère de province. Il est remarquable que, parmi les personnages engagés dans l'érection de ce temple, la plupart sont des proches du Bakufu de Kamakura, fidèles de Sanetomo, dont Matsuba Jirō Sukemune, devenu moine Zen sous le nom de Gyōen (?-1212-1257-?), lettré qui est resté ami avec Myōe et a été lié à Hatano Jirō (?-1212-1213-?), un frère de Hatano Yoshishige (?-1258), le grand protecteur de Dōgen que celui-ci pourrait avoir connu dès son retour de Chine.

14. Meigetsuki, III : 242 (Kangi 2, $9^{\mathrm{e}}$ mois, 19e jour). Cette fille adoptive, Zenkō no himegimi, est décédée à l'âge de 30 ans. Kobata est un site de la banlieue méridionale de Kyōto, où Dōgen semble avoir vécu une partie de sa jeunesse, dans la résidence des Matsudono, et près duquel il s'est établi peu de temps après son retour de Chine en 1227, à Fukakusa, partageant à cette époque son temps entre le Kenninji et Fukakusa. 
1233) et qui est décrite comme résidant dans le quartier de Shirakawa à Kyōto. Un argument vient renforcer ce sentiment : Dōgen vient s'installer à Fukakusa, dans l'enceinte du Gokurakuji, dans les années 1230-1231. Or, ce domaine appartenait à cette époque à Motomichi, personnage très proche de Michichika de son vivant, et par ailleurs grand protecteur de Myōe. On comprendrait en effet que la puissante donatrice, préoccupée du salut posthume de son fils Chikahira, se soit tournée à cette époque vers Dōgen après la mort de Myōe survenue en 1232 puis celle de son mari l'année suivante, et peut-être en raison d'un réseau de connaissances communes ${ }^{15}$. L'absence de mention du nom de cette moniale dans les sources autres que celles du milieu de Dōgen ne pourrait-elle se comprendre en raison du fait qu'on aurait spécialement choisi pour elle celui du moine Zhengjue (jap. Shōgaku) (1091-1157), vénéré dans le Sōtō et notamment par Dōgen comme le réformateur de cette école en Chine?

Cette hypothèse hautement vraisemblable d'identification, car la plus cohérente à ce jour, concourt à montrer que les deux moines sensiblement contemporains qu'étaient Myōe et Dōgen avaient des réseaux de patronage dans les milieux de la cour impériale de Kyōto et du Bakufu de Kamakura. Même si l'hypothèse se trouve un jour controuvée, on peut tout du moins avancer que ces deux personnages avaient dans tous les cas des appuis équivalents en puissance : on ne peut donc dire que ce qui différencie le premier et le second, qui seraient censés représenter respectivement l'ancien et le nouveau bouddhisme à l'époque, tiendrait à une allégeance à la cour ou au Bakufu, à un patronage laïc important ou encore à une absence de patronage. Au demeurant, ces notions d'ancien et de nouveau bouddhisme, après avoir été sérieusement remises en question, sont maintenant mises au second plan, voire abandonnées, par les meilleurs spécialistes pour envisager d'autres catégories et systèmes d'explication. On préfère actuellement définir le caractère médiéval du bouddhisme japonais, caractère qui en marquerait sa japonité, par le système combiné de l'exotérisme et de l'ésotérisme (kenmitsu taisei), avec des nuances plus ou moins prononcées selon les auteurs ${ }^{16}$. En l'occurrence, il semble que si ce qui différencie ces deux grands moines ne relève pas de leurs systèmes de protection laïque, c'est sur le plan de la pensée et des pratiques religieuses qu'il convient de le chercher : Myōe correspond exactement à ce système médiéval associant exotérisme et ésotérisme - il passe pour le fondateur de l'ésotérisme Kegon - ; Dōgen en revanche, en refusant d'intégrer l'ésotérisme au Zen, ainsi que le faisaient presque tous ses contemporains (Yōsai, Benni, Gyōyū), est peut-être le premier moine japonais à avoir proposé une forme de bouddhisme ayant entièrement évacué des éléments cérémoniels, cultuels et symboliques qui séduisaient tant la cour de Kyōto, et en conséquence une forme de bouddhisme " extramédiévale » : ceci expliquerait, en partie mais de façon suffisante, l'incompatibilité des formes de bouddhisme que Dōgen préconisait avec les milieux de Kyōto et son départ de cette ville pour la lointaine province d'Echizen, d'une part, l'intérêt des philosophes

15. Nakaseko Dōshō, Dōgen zeniji-den kenkyū, zoku [Recherches sur la biographie de Dōgen, Suite], Tōkyō, Kokusho kankōkai, 1997 : 254-256. Les autres hypothèses passées en revue par l'auteur semblent toutes fantaisistes et peu fiables.

16. Voir à ce sujets les discussions de : Taira Masayuki, "Nihon chūsei no shakai to bukkyō", "Société et bouddhisme dans le Moyen Âge japonais» et «Kuroda Toshio and the Kenmitsu taisei Theory », Japanese Journal of Religious Studies, 23 (2-4), 1996 ; Sueki Fumihiko, " A Reexamination of the Kenmitsu taisei Theory », in ibidem ; Sueki Fumihiko, "Annen : The Philosopher Who Japanized Buddhism », Acta Asiatica, 66, Buddhism in East Asia, The Tōhō Gakkai, Tōkyō, 1994 ; Sueki Fumihiko, Kamakura bukkyō keisei-ron - shisōshi no tachiba kara -, Études sur la formation du bouddhisme de Kamakura, du point de vue de l'histoire de la pensée, Hōzōkan, Kyōto, 1998. 
japonais du $\mathrm{XX}^{\mathrm{e}}$ siècle qui voient en lui le premier philosophe japonais, en raison du caractère purement exotérique de sa pensée, d'autre part.

Des quelques remarques que m'a inspirées la lecture du présent fragment du Journal des rêves de Myōe, aucune conclusion ne se dégage définitivement pour le moment. Néanmoins, j'espère qu'elles ont pu faire toucher du doigt le fait que l'identification précise de personnages qui apparaissent dans les documents n'est pas sans influer sur l'évaluation exacte qu'on est en mesure de faire concernant le patronage des activités et des fondations religieuses, ainsi que sur les liens qu'entretiennent entre eux les religieux, et partant sur le sens même des mouvements dont ils sont les proteurs et les initiateurs.

\section{Traduction}

« (236) La nuit du $22^{\mathrm{e}}$ jour du même mois, je fis un rêve :

Quittant Takao, je me mêlai à de nombreuses gens et leur donnai d'excellents médicaments qu'ils prirent.

La première année de l'ère Karoku (1225), le $16 \mathrm{du} 8^{\mathrm{e}}$ mois, à l'heure du tigre - on est la nuit du 15 -, on a transféré les augustes réceptacles - les déesses Byakkō et Zenmyō des pavillons central et de droite dans le sanctuaire. Le ciel s'était alors assombri ${ }^{17}$ depuis un moment. Quand la cérémonie fut finie, l'on s'en retourna ${ }^{18}$ dans les cellules. Il se mit alors à pleuvoir ${ }^{19}$. Le matin du jour suivant, une forte pluie éclata également, et la journée il venta violemment. Vers l'heure du mouton, on fit la dédicace dans le pavillon à prière, et les offrandes avec l'Avatamsakasütra en 80 volumes. Le maître de vœu, le religieux préfet monacal [Gyōkan], mit les auditeurs en rang. À ce moment-là, la pluie cessa quelques instants, le vent se calma quelque peu et l'on commença la cérémonie. Arrivé le moment de la partie des dieux ${ }^{20}$, il plut à nouveau et, dès qu'elle fut finie, le ciel se dégagea, laissant apparaître dans tout son éclat la lumière du soleil ${ }^{21}$.

(237) La nuit, je fis un rêve :

(a) Dans un pavillon, se trouvait feu le préfet monacal Dōchū et, de l'autre côté du mur, une femme qui paraissait être son auguste présence Maya. Le préfet monacal l'admonestait. Elle prit congé du préfet monacal pour se rendre ailleurs, en un endroit que, semble-t-il, elle fréquentait et où elle demeurait (= le Zenmyōji). Le préfet monacal l'admonesta à nouveau et lui donna des ordres.

(b) Je m'endormis à nouveau. [Je vis en rêve] feu le laïc entré en religion d'Izu [Fujiwara no Chikayasu] sous son aspect d'homme ordinaire. Je voulais $m$ 'en aller, mais il me retint à un endroit ${ }^{22}$ - c'est qu'il y avait une autre affaire - . Il me fit assoir dans la salle de garde située dans la résidence du Gouverneur de troisième rang ${ }^{23}$. Je vis alors sur un mur un portrait ${ }^{24}$ qui était suspendu et qui ressemblait à s'y méprendre à son modèle.

Je me réjouis du texte des instructions ${ }^{25}$.

- Ibidem. 》

17. Lire kumoru 喑, être nuageux, au lieu de haru 晴, être dégagé.

18. Lire kaheru 還, s'en retourner, au lieu de oku 置, poser.

19. Lire furu 降, pleuvoir, au lieu de fukaki 深, profond.

20. La partie des dieux (jinbun 神分) correspond au moment où, dans une cérémonie, on récite divers textes, comme le Sūtra de la quintessence de la sapience (Hannyashingyō 般若心經), à destination des divinités.

21. Lire nikkō日光, lumière du soleil, au lieu de Byakkō白光.

22. Lire aru issho 或一所, en un endroit, et non fusu tokoro 伏又所, être allongé (cf. supra).

23. Kō no sanmi dono 守三位殿.

24. De Chikayasu?

25. kyōkunjō 教訓狀. 


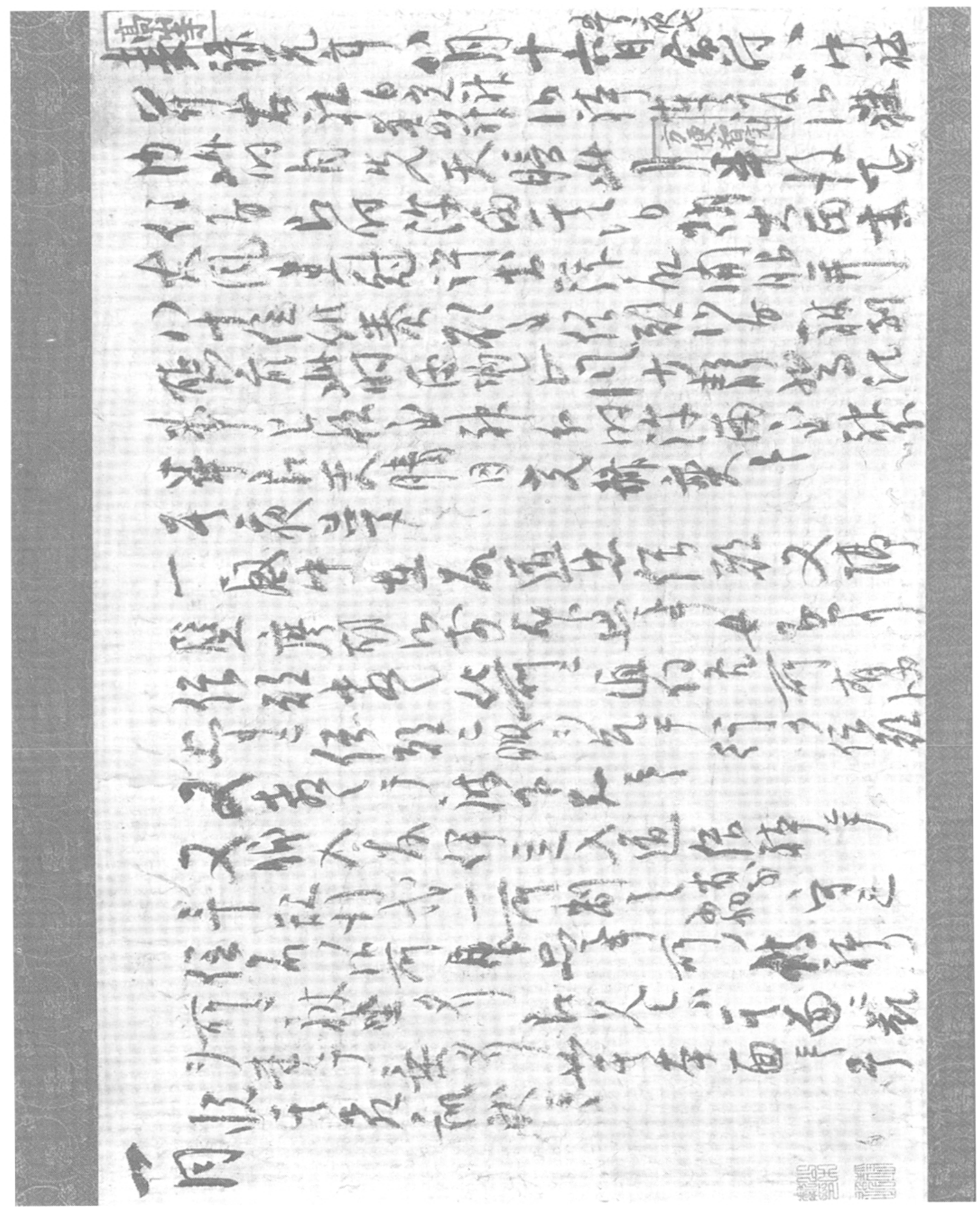

Myōe, Muki, daté de 1225 , encre sur papier, 33,7 ×55,3 cm (The Harvard University Art Museum, Cambridge, Massachusetts) 


\title{
Abréviations
}

MSS : “ Myōe shōnin shiryō 明惠上人資料, Matériaux relatifs au révérend Myōe », in Kōzanji shiryō sōsho 高山寺資料叢書, Collection de matériaux du Kōzanji, Tōkyō daigaku shuppankai, Éditions de l’Université de Tōkyō, 1971.

ZGSRJ : Zoku gunsho ruijū 續群書類從.

\begin{abstract}
Some Remarks on the Harvard Fragment of the Dreams Record of Myōe (1173-1232)

In the present paper, I analyse a fragment of the Dream Diary of Myōe (1173-1232), conserved at the Harvard University Art Museum in Cambridge, Mass. This fragment seems to be a better, that is earlier, version of the Dream Diary than the one that has been conserved in Kōzanji monastery, and that has already been published. Here we present a new translation and analysis of it. I refer to the hypothesis concerning the identification of the personnage of Kō no sanmi no tsubone, who seems to be alluded to in this fragment, and who is a great patron of Myōe and Kōzanji. This identification is not only interesting from the historical point of view, but also provides new perspectives on the relationship between Myōe, traditionally considered as representative of ancient Buddhism, and Dōgen, who is thought to be representative of the so-called new Buddhism of this period : in fact, both communities seem to have had the same patron. The discovery of historical details permits us to reconsider pertinent paradigms and categories to define and analyse medieval Buddhism as japanised Buddhism.
\end{abstract}

Lignée généalogique I

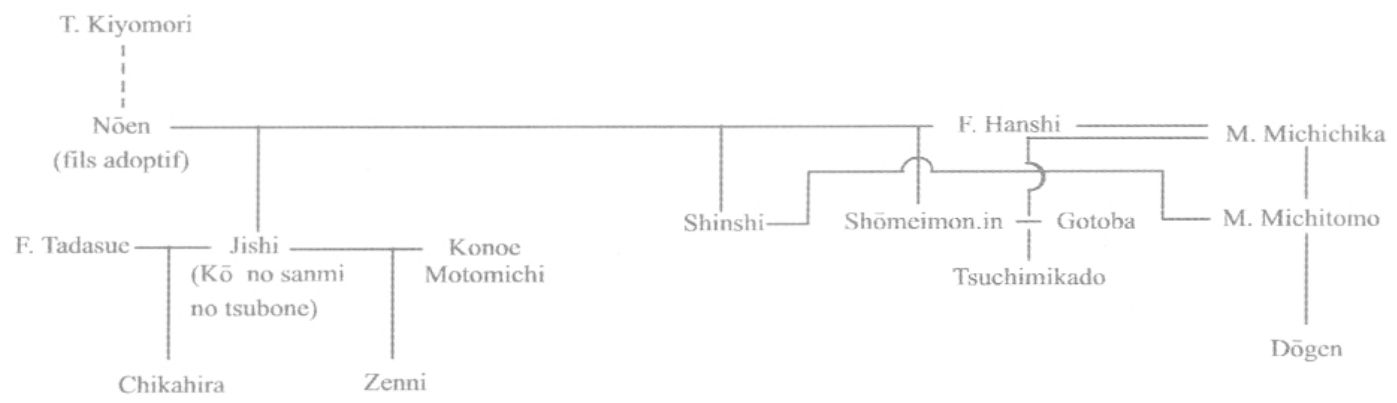

Lignêc généalogique 2

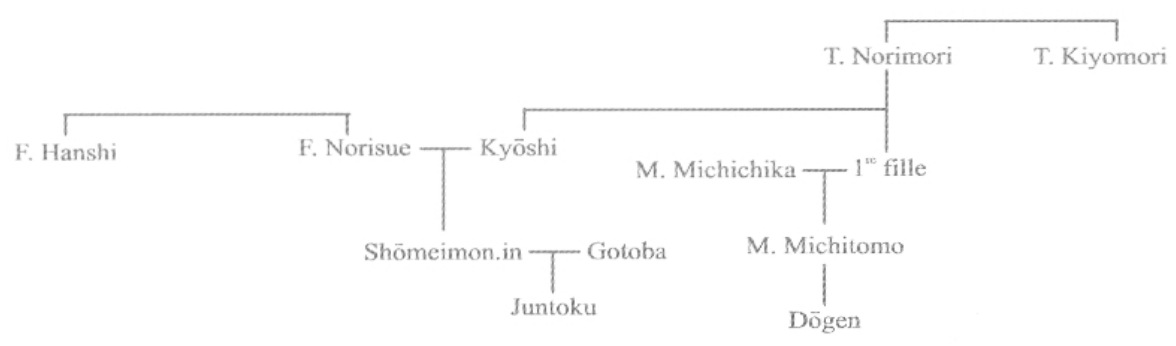

